\title{
The Amorphous Spin-1 Ising Model on the Bethe Lattice
}

\author{
E. AlbayraK* \\ Erciyes University, Department of Physics, 38039, Kayseri, Turkey
}

(Received May 17, 2018; in final form October 26, 2018)

\begin{abstract}
The coordination numbers $z=3,4$ and 6 corresponding to the honeycomb, square, and simple cubic lattices, respectively, are randomly taken with probabilities $p, q, r$ between the nearest-neighbors of spin-1 atoms on the Bethe lattice to simulate amorphous materials. The exact recursion relations are employed to obtain the orderparameters with the implementation of random coordination numbers in the standard-random approach. The phase diagrams are obtained by varying the temperature for given values of crystal field, coordination numbers, and probabilities. It is found that the phase lines consist of either second- or first-order phase transitions and secondand first-order phase transitions combined at the tricritical points for appropriate values of our parameters.
\end{abstract}

DOI: 10.12693/APhysPolA.134.1176

PACS/topics: randomness, coordination number, Bethe lattice, spin-1

\section{Introduction}

The amorphous material is a type of non-equilibrium material whose atomic arrangement is more like liquid with no long-range periodicity. They can be produced by rapid solidifications to freeze the liquid structure of the alloy melt, or other methods can also be used that can mix atoms to acquire a disordered state. After the rapid solidification, the atoms freeze in such a way that having different numbers of the nearest-neighbors (NN), i.e. coordination numbers (CN's). Therefore, the subject of this work is to consider the effects of randomness in the CN's of a simulated alloy on the Bethe lattice (BL). As a result, many properties of the material can change which may also include the changes in electric and magnetic behaviors.

The spin-1 Ising model is the lowest spin model including the crystal field effects called as the Blume-Capel (BC) model and the literature is very vast for it. So far only a few works have considered the randomness in the CN's: The amorphous spin-1 Ising model with effective $\mathrm{CN}$ was studied using the effective-field theory with correlations [1], the zero-temperature hysteresis in the random-field Ising model on a BL where a fraction $c$ of the sites have $\mathrm{CN}$ of $z=4$ while the remaining fraction $1-c$ have $z^{\prime}=3[2]$ and the last work was also carried out on the BL by considering the CN's in doubles, i.e. $z$ and $z^{\prime}$, with $z \neq z^{\prime}=3,4$ and 6 by using the exact recursion relations (ERR) in the standard-random distribution [3].

In this work, we take the randomness in the CN's as triplets, i.e. $z=3$ with probability $p, z=4$ with probability $q$ and $z=6$ with probability $r$ and obeying the normalization condition $p+q+r=1$. Therefore, the amorphousness of this work is more serious than the work taking the CN's as doubles [3]. Thus, it deserves to be investigated. We consider the spin-1 $\mathrm{BC}$ model on the $\mathrm{BL}$

*corresponding author; e-mail: albayrak@erciyes.edu.tr in terms of ERR's when the number of the NN's of each spin changes randomly. For this randomization, the CN's $z=3,4$ and 6 are considered as a triplet which are randomly varied on the shells of the BL. The phase diagrams are calculated by studying the second- and first-order phase transition lines and the tricritical points (TCP).

The remainder of this work is set up as follows: The next section is devoted to the formulation of spin- $1 \mathrm{BC}$ model in terms of ERR's and the procedure of randomness of the CN's in triplets in the standard-random approach [4]. The phase diagrams are illustrated and possible conclusions are made in the last section.

\section{The formulation}

In this work, the usual Blume-Capel Hamiltonian including the bilinear interaction parameter $J$ and the crystal field term $D$ is considered which is given as

$$
\mathcal{H}=-J \sum_{<i, j>} \sigma_{i} \sigma_{j}-D \sum_{i} \sigma_{i}^{2},
$$

where $\sigma_{i}$ is an Ising spin of spin- 1 with the values \pm 1 and 0 . Thus, this model is a three state and two orderparameters, dipolar and quadrupolar moments, system. In this work, the ERR's are employed to obtain all the thermodynamic functions of the system.

It should be noted that the ERR's were already calculated in detail in some previous studies such as the [5] (and also the references therein), therefore, they are just taken from there without giving any details. Since spin1 model has three states, therefore, one gets two ERR's with $z$ NN's. They are calculated as

$X_{n}=\frac{g_{n}(+1)}{g_{n}(0)}=\frac{\mathrm{e}^{\beta^{\prime}(1+d)} X_{n-1}^{z-1}+\mathrm{e}^{\beta^{\prime}(-1+d)} Y_{n-1}^{z-1}+1}{\mathrm{e}^{\beta^{\prime} d} X_{n-1}^{z-1}+\mathrm{e}^{\beta^{\prime}} Y_{n-1}^{z-1}+1}$,
$Y_{n}=\frac{g_{n}(-1)}{g_{n}(0)}=\frac{\mathrm{e}^{\beta^{\prime}(-1+d)} X_{n-1}^{z-1}+\mathrm{e}^{\beta^{\prime}(1+d)} Y_{n-1}^{z-1}+1}{\mathrm{e}^{\beta^{\prime} d} X_{n-1}^{z-1}+\mathrm{e}^{\beta^{\prime} d} Y_{n-1}^{z-1}+1}$,

where $d=D / J$ is the reduced crystal field, $\beta^{\prime}=\beta J=$ $J / k T$ is the reduced temperature and $k$ is the Boltzmann constant. $n$ is the number of the shells of the BL and it is taken to be infinite in the thermodynamic limit. 
The BL is constructed by picking a central spin to which $z=3,4$ or 6 NN's with probabilities $p, q, r$ are connected randomly. Then, the NN spins are again connected with $z=3$, 4 or 6 NN's again with probabilities $p, q, r$ randomly. Then the same procedure is repeated for the next NN's and so on to infinity. Thus the number of the NN's, i.e. CN's, are distributed throughout the $\mathrm{BL}$ according to

$$
P\left(z_{i}\right)=p \delta\left(z_{i}-z\right)+q \delta\left(z_{i}-z^{\prime}\right)+r \delta\left(z_{i}-z^{\prime \prime}\right),
$$

where $p$ is the probability of a spin having $z=3, q$ is the probability of a spin having $z^{\prime}=4$ and $r$ is the probability of a spin having $z^{\prime \prime}=6$. The regular case of $\mathrm{BC}$ is regained if any of the two probabilities are set equal to zero.

In order to implement the random distributions of CN's $z, z^{\prime}$ and $z^{\prime \prime}$ in the standard-random approach [4], the ERR's, i.e. Eq. (2), should be rewritten by the application of Eq. (3) as

$$
\begin{aligned}
& X_{R}=p X_{n z}+q X_{n z^{\prime}}+r X_{n z^{\prime \prime}}, \\
& Y_{R}=p Y_{n z}+q Y_{n z^{\prime}}+r Y_{n z^{\prime \prime}},
\end{aligned}
$$

where $\left(X_{n z}, Y_{n z}\right)$ is given in Eq. (2). $\left(X_{n z^{\prime}}, Y_{n z^{\prime}}\right)$ and $\left(X_{n z^{\prime \prime}}, Y_{n z^{\prime \prime}}\right)$ are for $z=z^{\prime}$ and $z=z^{\prime \prime}$, respectively. $\left(X_{R}, Y_{R}\right)$ are the ERR's for the randomly distributed CN's on the BL.

The order-parameters of our model, magnetization and quadrupole moment, are given in terms of the ERR's for the $z$ NN's as

$$
\begin{aligned}
& M_{z}=\left\langle S_{i}\right\rangle=\frac{\mathrm{e}^{\beta^{\prime} d} X_{R}^{z}-\mathrm{e}^{\beta^{\prime} d} Y_{R}^{z}}{\mathrm{e}^{\beta^{\prime} d} X_{R}^{z}+\mathrm{e}^{\beta^{\prime} d} Y_{R}^{z}+1}, \\
& Q_{z}=\left\langle S_{i}^{2}\right\rangle=\frac{\mathrm{e}^{\beta^{\prime} d} X_{R}^{z}+\mathrm{e}^{\beta^{\prime} d} Y_{R}^{z}}{\mathrm{e}^{\beta^{\prime} d} X_{R}^{z}+\mathrm{e}^{\beta^{\prime} d} Y_{R}^{z}+1} .
\end{aligned}
$$

Similar equations hold for $\left(M_{z^{\prime}}, Q_{z^{\prime}}\right)$ and $\left(M_{z^{\prime \prime}}, Q_{z^{\prime \prime}}\right)$ corresponding to the $z^{\prime}$ and $z^{\prime \prime}$ NN's, respectively. Finally, the net magnetization and net quadrupolar moments are obtained from

$$
\begin{aligned}
& M_{\text {net }}=p M_{z}+q M_{z^{\prime}}+r M_{z^{\prime \prime}}, \\
& Q_{\text {net }}=p Q_{z}+q Q_{z^{\prime}}+r Q_{z^{\prime \prime}} .
\end{aligned}
$$

The next section is devoted to obtaining of the values of ERR's by iteration to study the thermal variations of the order-parameters to obtain the phase diagrams.

\section{Results and conclusions}

Before going into the calculations of the phase diagrams, let us first discuss the general case of triple random $\mathrm{CN}$ distribution that reduces to some specific models for some special values of the probabilities. When one of the probabilities, i.e. $p, q, r$ are taken to be equal to one and the others set to zero, the model reduces to the wellknown BC model, with $z=3,4$ and 6, respectively [5]. It should be mentioned that the phase lines only consist of second-order phase transition temperatures, $T_{c}$, for $z=3$, the phase lines consist of $T_{c}$ and the lines of first-order phase transition temperatures, $T_{t}$, combined at a tricritical point (TCP) for $z=6$, and there are some controversial results for $z=4$ whether it is a $z=3$ or $z=6$ type. The cases with $p=0$ and $(q, r)$ varied, $q=0$ and $(p, r)$ varied and $r=0$ and $(p, q)$ varied correspond to the double random variations of CN's with $(z=3$, $4),(z=3,6)$ and $(z=4,6)$ cases, respectively [3]. Thus, this work is the most serious random case of the CN's so far which maybe extended to other CN's such as $z=8,12$ and so on very easily by using the approach given in here.

Thus, in this work we study the thermal variations of the order-parameters to calculate the phase diagrams on the planes of (probability, $k T / J)$ and $(d, k T / J)$ for given $d$ and probabilities $p, q, r$ between 0 and 1 , respectively. To be more consistent, we did not study the cases when one of the probability is equal to one or zero, since those cases were already studied as mentioned above. In the phase diagrams, the solid and dashed lines indicate the $T_{c^{-}}$and $T_{t^{-}}$-lines, respectively. The TCP's at which the $T_{c^{-}}$and $T_{t^{-}}$lines are connected are indicated with solid squares.

The first phase diagrams are studied on the $(d, k T / J)$ planes with $r=1-p-q$ when $p=0.1,0.3,0.5,0.7$ and 0.8 for given values of $q$ as labeled on the lines with the increment of $\triangle q=0.1$. In this range, some probability values are scanned and presented in Fig. 1a-d. As seen, the phase lines always consist of $T_{c^{-}}$and $T_{t}$-lines combined at TCP's. The lines starts as $T_{t}$-lines at very low temperatures and low negative $d$ 's, as $d$ becomes more positive these lines terminate at higher temperatures at the TCP's from where the $T_{c}$-lines emerge which increase in temperature as $d$ increases further. This behavior is a well-known behavior of the spin-1 BC model. The TCP's lie along the left-slanted straight lines which means that as $q$ increases or $r$ decreases, they move to lower temperatures and to lower negative $d$ 's. This is clear, since $z=6$ is larger than $z=4$, i.e. as $q$ increases and $r$ decreases the critical temperatures are lowered which is a common characteristic of the Ising models. The same also occurs if $p$ increases while $q=0.8-p-r$ is varied, since $z=3$ is the lowest $\mathrm{CN}$ of this work. It should be also noted that these phase lines are qualitatively similar with the phase lines of the works $[1,3]$, where CN's are varied in doubles only. It should also be emphasized that the TCP's must satisfy the universal relation $D /(z J)=0.47$, see for example [6]. In our calculation, in terms of the probabilities and CN's it should be calculated according to the formula $D /\left[\left(p \times z+q \times z^{\prime}+r \times z^{\prime \prime}\right) J\right]$ which yields a value of about 0.48 which is very close to this universal value.

The rest of the phase diagrams are calculated on possible probability versus temperature planes for given $d$ 's. The obtained phase diagrams are qualitatively very similar to each other, i.e. at higher $d$ 's only straight $T_{c}$-lines are seen, then for some range of negative $d$ values, first $T_{c}$-lines appear at low probabilities which terminate at TCP's as probabilities increase from where the $T_{t}$-lines appear which terminate at possible highest probability values and for further negative $d$ 's they are always in the form of $T_{t}$-lines. 

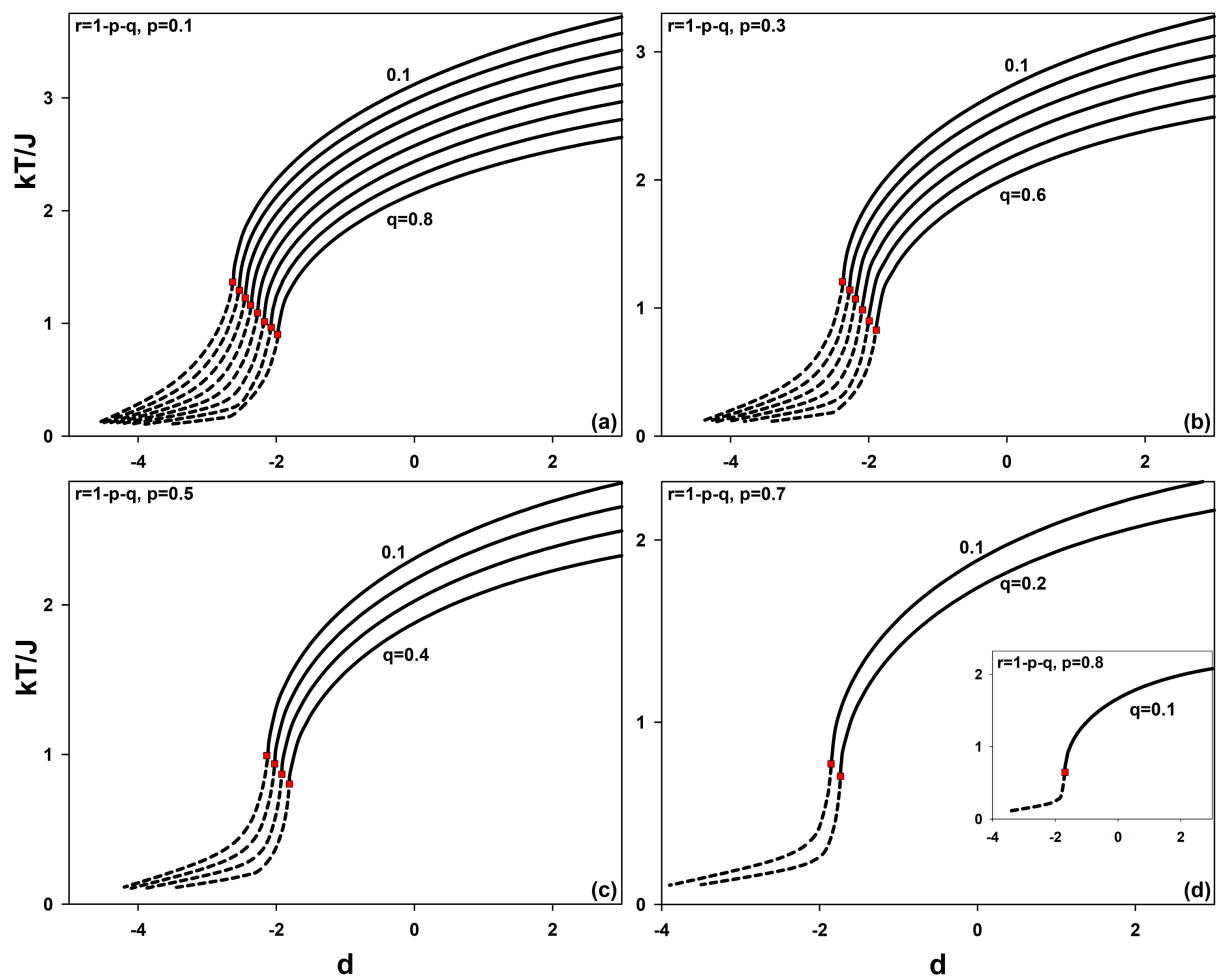

Fig. 1. The phase diagrams on the $(d, k T / J)$ planes for given values of the probabilities $p, q, r$ between 0.1 and 0.8 with the increments of 0.1 when $r=1-p-q$; (a) $p=0.1,0.1 \leq q \leq 0.8$, (b) $p=0.3,0.1 \leq q \leq 0.6$, (c) $p=0.5$, $0.1 \leq q \leq 0.4$ and (d) $p=0.7,0.1 \leq q \leq 0.2$ and in the inset $p=0.8, q=0.1$.
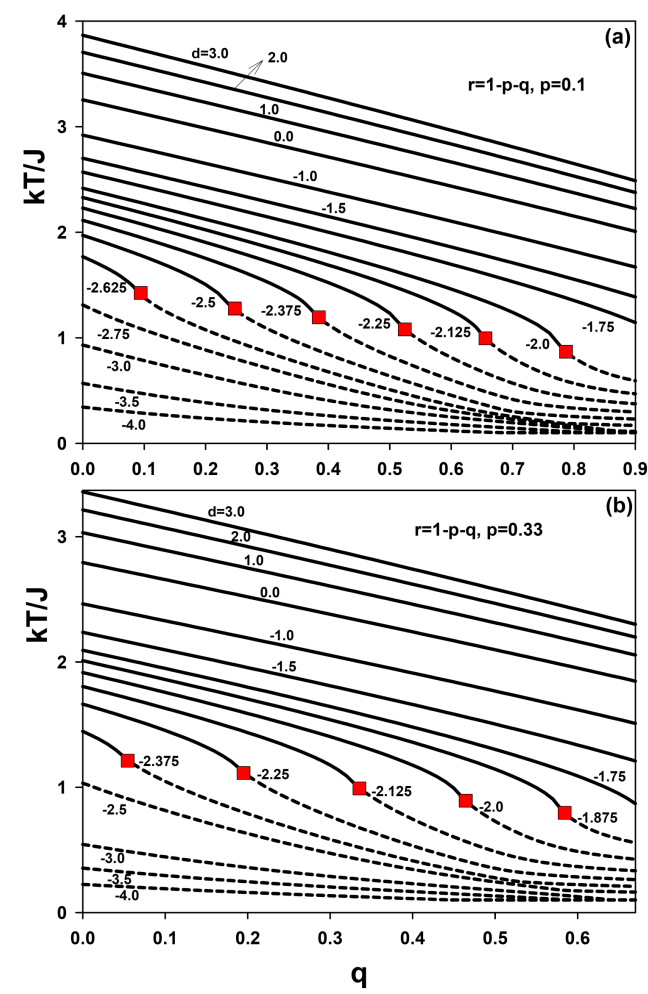

Fig. 2. The phase diagrams on the $(q, k T / J)$ planes for given values of $d$ with $r=1-p-q$; (a) $p=0.1$ and (b) $p=0.33$.
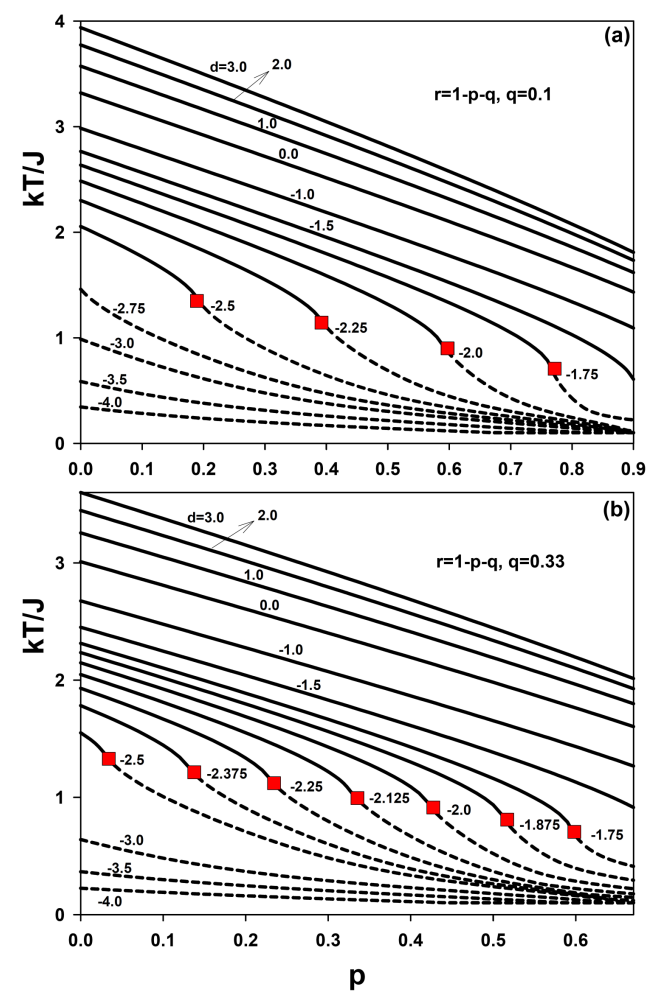

Fig. 3. The phase diagrams on the $(p, k T / J)$ planes for given values of $d$ with $r=1-p-q$; (a) $q=0.1$ and (b) $q=0.33$. 

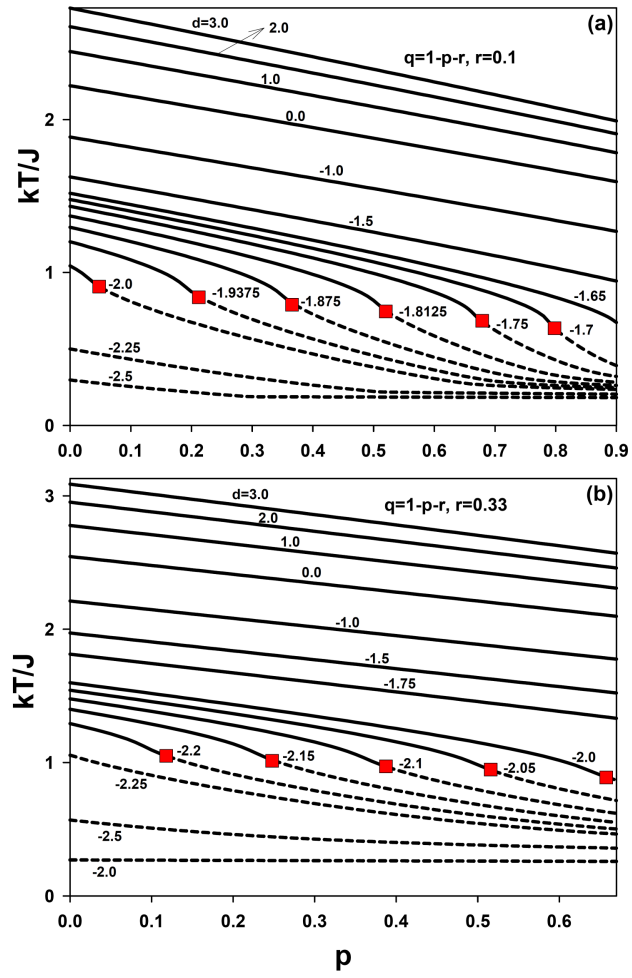

Fig. 4. The phase diagrams on the $(p, k T / J)$ planes for given values of $d$ with $q=1-p-r$; (a) $r=0.1$ and (b) $r=0.33$.

Figure 2a,b are the phase diagrams obtained on the $(q, k T / J)$ on the planes for $p=0.1$ and 0.33 when $r=1-$ $p-q$, respectively. The temperatures of the critical lines decrease as $q(r)$ increases (decreases). This is obvious since 6 is larger $\mathrm{CN}$ than 4 , thus, higher $\mathrm{CN}$ means higher temperatures.

Figure 3a,b are the phase diagrams obtained on the $(p, k T / J)$ on the planes for $q=0.1$ and 0.33 when $r=$ $1-p-q$, respectively. The temperatures of the critical lines again decrease as $p(r)$ increases (decreases). This is again obvious since 6 is larger $\mathrm{CN}$ than 3 .
The final phase diagrams, Fig. 4a,b, are also obtained on the $(p, k T / J)$ on the planes for $r=0.1$ and 0.33 when $q=1-p-r$, respectively. The temperatures of the critical lines again decrease as $p(q)$ increases (decreases), since now 4 is larger $\mathrm{CN}$ than 3 . They are again very similar with the phase diagrams given in [3].

In concluding this work, we can summarize our findings as follows: The obtained phase diagrams are qualitatively similar with each other, they only change quantitatively. They always consist of the $T_{c^{-}}$and $T_{t}$-lines combined at TCP's on the $(d, k T / J)$ planes for all given probability values $p, q, r$. It seems that the behavior of critical points and lines are affected by $d$ but their places are determined by the values of the probabilities in addition to $d$. The phase diagrams on the probability versus temperature planes consist of only $T_{c}$-lines at higher values of $d$, the $T_{c^{-}}$and $T_{t}$-lines combined at TCP's for some negative range of $d$ values and only $T_{t}$-lines for low enough negative $d$ values. Our obtained value of $D / z J$ is about 0.48 which is very close to the universal value. It should be noted that if one increases the CN's further, it seems that it would only change the temperatures of the critical lines, that is the qualitative behavior should remain the same as with the findings of this work.

\section{References}

[1] T. Kaneyoshi, Phys. Status Solidi B 146, 253 (1988).

[2] P. Shukla, D. Thongjaomayum, J. Phys. A Math. Theor. 49, 235001 (2016).

[3] E. Albayrak, J. Supercond. Nov. Magn. 31, 3595 (2018).

[4] E. Albayrak, Solid State Commun. 159, 76 (2013); E. Albayrak, Physica B 531, 70 (2018).

[5] E. Albayrak, Physica A 390, 1529 (2011); E. Albayrak, Int. J. Mod. Phys. B 26, 1250031 (2012); E. Albayrak, Commun. Theor. Phys. 68, 361 (2017); E. Albayrak, S. Akkaya, T. Cengiz, J. Magn. Magn. Mater. 321, 108 (2009).

[6] Proc. 5th Int. Conf. on Physics of Magnetic Materials, Eds. W. Gorzkowski, M. Gutowski, H.K. Lachowicz, H. Szymczak, World Sci., 1991, p. 45. 